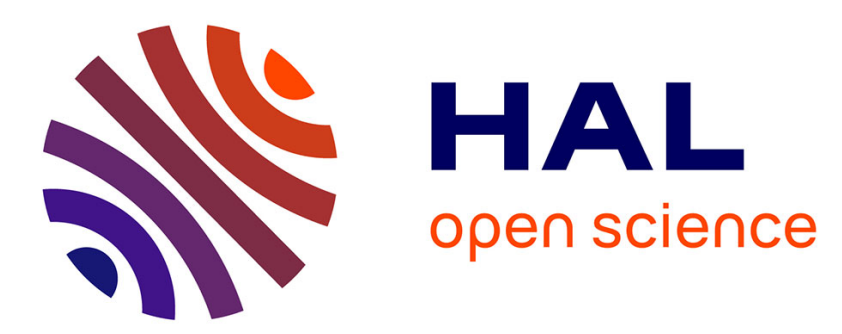

\title{
Building Portable Thread Schedulers for Hierarchical Multiprocessors: the BubbleSched Framework
}

\author{
Samuel Thibault, Raymond Namyst, Pierre-André Wacrenier
}

\section{To cite this version:}

Samuel Thibault, Raymond Namyst, Pierre-André Wacrenier. Building Portable Thread Schedulers for Hierarchical Multiprocessors: the BubbleSched Framework. EuroPar, Aug 2007, Rennes, France. 10.1007/978-3-540-74466-5_6. inria-00154506

\section{HAL Id: inria-00154506 \\ https://hal.inria.fr/inria-00154506}

Submitted on 13 Jun 2007

HAL is a multi-disciplinary open access archive for the deposit and dissemination of scientific research documents, whether they are published or not. The documents may come from teaching and research institutions in France or abroad, or from public or private research centers.
L'archive ouverte pluridisciplinaire HAL, est destinée au dépôt et à la diffusion de documents scientifiques de niveau recherche, publiés ou non, émanant des établissements d'enseignement et de recherche français ou étrangers, des laboratoires publics ou privés. 


\title{
Building Portable Thread Schedulers for Hierarchical Multiprocessors: the BubbleSched Framework
}

\author{
Samuel Thibault, Raymond Namyst, and Pierre-André Wacrenier \\ INRIA Futurs - LABRI - 351 cours de la libération - 33405 Talence cedex, France \\ \{thibault, namyst, wacrenier\}@labri.fr
}

\begin{abstract}
Exploiting full computational power of current more and more hierarchical multiprocessor machines requires a very careful distribution of threads and data among the underlying non-uniform architecture. Unfortunately, most operating systems only provide a poor scheduling API that does not allow applications to transmit valuable scheduling hints to the system. In a previous paper [10], we showed that using a bubble-based thread scheduler can significantly improve applications' performance in a portable way. However, since multithreaded applications have various scheduling requirements, there is no universal scheduler that could meet all these needs. In this paper, we present a framework that allows scheduling experts to implement and experiment with customized thread schedulers. It provides a powerful API for dynamically distributing bubbles among the machine in a high-level, portable, and efficient way. Several examples show how experts can then develop, debug and tune their own portable bubble schedulers.
\end{abstract}

Keywords: Threads, Scheduling, Bubbles, NUMA, SMP, Multi-Core, $S M T$.

\section{Introduction}

Both AMD and INTEL now provide quad-core chips and are heading for 100cores chips. This is, in the low-end market, the emerging part of a deep trend, in the scientific computation market, towards more and more complex machines (e.g. Sun WildFire, SGI Altix, Bull NovaScale). Such large sharedmemory machines are typically based on Non-Uniform Memory Architectures (NUMA). Recent technologies such as Simultaneous Multi-Threading (SMT) and multi-core chips make these architectures even more hierarchical.

Exploiting these machines efficiently is a real challenge, and a thread scheduler is faced with dilemmas when trying to take into account the memory hierarchy and the CPU utilization simultaneously. On NUMA machines for instance, threads should generally be scheduled as close to their data as possible, but bandwidth-consuming threads should rather be distributed over different chips. The core scheduler of operating systems can often be influenced, but it misses the precise application behavior: for instance, adaptively-refined meshes entail 
very irregular and unpredictable behavior. A good solution would be to let application programmers take control of the scheduling, but writing a whole scheduler for hierarchical machines is a very difficult task.

In a previous paper [10], we introduced the bubble scheduling concept that helps to express the inherent parallel structure of multithreaded applications in a way that can be efficiently exploited by the underlying thread scheduler. Bubbles are abstractions to group threads which "work together" in a recursive way. The first proof-of-concept implementation of our bubble scheduler was featuring a generic hard-coded scheduler. However, applications may have different scheduling requirements and thus may attach different semantics to bubbles, enforcing memory affinity or emphasizing a high frequency of global synchronization operations for instance. Obviously, no generic scheduler can meet all these needs. In this paper, we present BubbleSched, a framework designed to ease the development and the evaluation of customized, high-level thread schedulers.

\section{On the Design of Thread Schedulers}

Designing a thread scheduler for hierarchical machines is complex because it means finding an application-specific compromise between lots of constraints: favoring affinities between threads and memory, taking advantage of all computational power, reducing synchronization cost, etc.

\subsection{What Input Can a Scheduler Expect?}

To make appropriate decisions at execution time, a thread scheduler can combine a number of parameters to evaluate the goodness of each potential scheduling action. These parameters can be collected from several places, at different times.

At runtime, some useful knowledge about the target machine can be discovered. The scheduler can not only get the number of processors but also the architecture hierarchy: how processors and memory banks are connected, how cache levels are shared between processors, etc. Moreover, indication about how well the threads are using the underlying processors can be fetched from performance counters. Some NUMA chips can also report the ratio of remote memory accesses. The scheduler can hence check whether threads and data are properly localized, or enable automatic migration policies [6].

The compiler can also provide information about the application behavior. Data access patterns may be analyzed [7] and used to choose runtime allocation policies. An OpenMP compiler can sometimes accurately estimate the amount of data shared by the threads involved in the same parallel section.

Last but not least, programmers can also provide relevant information about the application behavior: how threads will mostly access data, what threads are I/O-bounded, etc.) Such information can help the scheduler to find a good tradeoff regarding the co-location of threads and data, and more generally can help to determine the combination of scheduling policies that will perform best. 
To sum it up, a lot of information is at the scheduler's disposal or can be collected at run time. Programmers can sometimes even provide additional scheduling hints. All we need is a reasonable way to pass this information from the programmer to the scheduler.

\subsection{On the Importance of Scheduling Guidance}

Opportunistic policies based on Self-Scheduling [3 are the most natural approaches regarding thread scheduling. They use a centralized list of ready threads (FrEeBSD 4, LinUX 2.4, Windows 2K) or a distributed one (FreEBSD 5, IRIX, LinUX 2.6) associated to load balancing mechanisms. Such an approach scales and adapts to new workloads, but it does not use affinity information from the compiler or the programmer, and thus can not achieve best performance.

For very regular problems, a predetermined approach can be used. The idea is to compute a priori a good distribution of threads and data that will be enforced during execution. The machine being dedicated to the application, thread scheduling can be fully controlled by binding exactly one kernel thread to each processor. This approach, used in the PASTIX solver for sparse linear systems [5], gives excellent performance for regular problems, but as soon as the solving time depends on the data or intermediate results, behavior prediction fails and performance may actually get worse than by using an opportunistic approach.

An intermediate approach is based on negotiation. Some language extensions such as OPEnMP, High Performance Fortran (HPF) or Unified Parallel C (UPC) let programmers write parallel applications by simply annotating the source code. The distribution and scheduling decisions then belong to the compiler. Such extensions get good performance by exploiting information from programmers, but the expressiveness is limited to "Fork-Join" decomposition schemes, and programmers can not express unbalanced parallelism for instance.

Negotiation yet appears to be a promising approach, because programmers just have to give suggestions and clues, sometimes indirectly, about the behavior of the threads. However, the runtime system has only little control over the operating system's thread scheduler. We believe that a good approach is to extend the scheduler interface so as to allow applications to transmit scheduling hints that will persist inside the scheduler during the lifetime of the threads.

\subsection{Towards a Toolbox for Developing Thread Schedulers}

In [10], we proposed a model that allows programmers to model the relationships between threads using nested sets called bubbles. Figure 1 illustrates this: four threads are grouped as pairs in bubbles (e.g. they work on the same data), which are themselves grouped along another thread in a larger bubble. This lets express relations like data sharing, collective operations, or more generally a particular scheduling policy need (serialization, gang scheduling, etc.) We also automatically model hierarchical machines with a hierarchy of runqueues. To each component of each hierarchical level of the machine is associated one runqueue: one per logical processor, core, chip, NUMA node, and one for the 

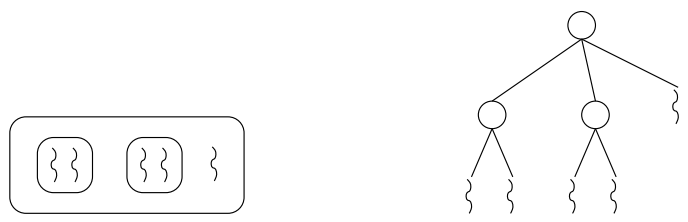

Fig. 1. Expressing thread relationships: graphical and tree-based representations

whole machine. Our ground scheduler then uses a hierarchical Self-Scheduling algorithm. Idle processors scan all runqueues that span them, and executes the first thread that they find, from bottom to top. For instance, if the thread is on a runqueue that represents a chip, it may be run by any processor of this chip.

\section{BubbleSched: A Framework for Building Portable Schedulers}

To tackle the delicate issue of scheduling an application on hierarchical machines in an efficient, flexible and portable way, we propose a new platform for easily writing customized schedulers, based on our high-level bubble abstractions.

Our platform allows programmers of specialized scientific libraries or parallel programming environments to easily write thread schedulers for various application classes on these machines. It provides a high-level API for writing powerful and portable schedulers that manipulate threads grouped into bubbles, as well as tracing tools to help analyzing the dynamic behavior of these schedulers. Programmers can hence focus on algorithmic issues rather than on technical details.

\subsection{An API for Writing Scheduling Strategies}

By default, bubbles are placed on the machine runqueue upon start-up. In the previous example of Figure 1 running on a bi-dual-core machine, this leads to Figure 2(a): the bubble hierarchy is kept at the top of the hierarchy of runqueues. Such a distribution permits the use of all processors of the machine, since all of them have the opportunity to run any thread. This does not however take affinities between threads and processors into account, since no relation between them is used. On the contrary, Figure 2(b) shows how threads can be spatially distributed in a very affinity-aware way. However, if some threads sleep, the corresponding processors become idle, resulting to a partial CPU usage.

We designed a programming interface [9] for manipulating bubbles and threads among the runqueues so as to achieve compromises between distributions of Figures 2(a) and 2(b). Threads and bubbles are equally considered as entities, while bubbles and runqueues are equally considered as scheduling holders, so that we end up with entities (threads or bubbles) that we can schedule on holders (bubbles or runqueues). Primitives are then provided for manipulating entities in 


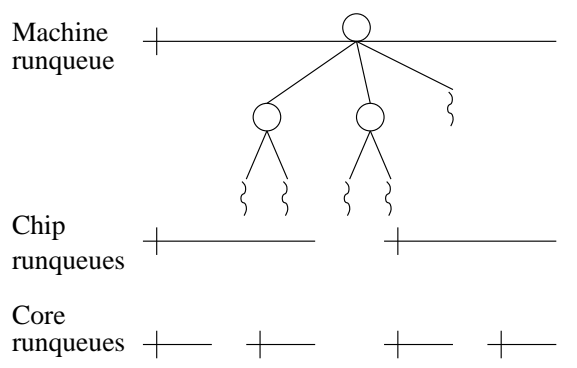

(a) Good CPU usage, poor affinity care.

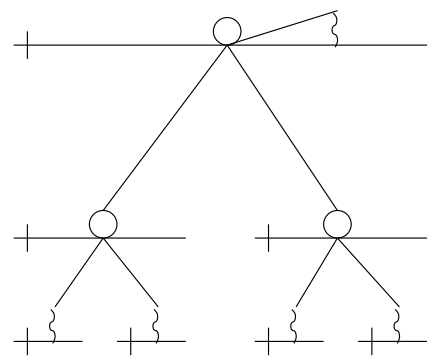

(b) Distributed CPU usage, good affinity care.

Fig. 2. Possible distributions of threads and bubbles among the machine.

holders. Runqueues can be accessed through vectors, and can be walked thanks to "father" and "son" pointers. Some functions permit to gather statistics about bubbles so as to take appropriate decisions. This includes the total number of threads and the number of running threads, but also the accumulated expected and current $\mathrm{CPU}$ computation time or memory usage, or the cache miss rates. To handle concurrency, one can use some fine-grain locking functions, but since bubble scheduling decisions are generally rather medium-term oriented, one can also just use a coarse-grain function which locks a whole sub-part of the machine (runqueues and bubbles). Once locked, one can enumerate entities held by bubbles and runqueues, and redistribute them at will. This permits to elaborate complex manipulations without having to care about locking.

Writing a scheduler actually reduces to writing some hook functions. The bubble_schedule() hook is called when the ground Self-Scheduler encounters a bubble while searching for the next thread to execute. By default, it just finds a thread in the bubble (or its sub-bubbles) and switches to it. The bubble_tick() hook is called when a time-slice for a bubble expires, permiting periodic operations on bubbles. Of course, mere "daemon" threads can also be started to perform background operations. Programmers may hence manipulate threads with a high level of abstraction by deciding the placement of bubbles on runqueues.

\subsection{Implementation Examples of Scheduling Algorithms}

Our programming interface, though quite simple, permits to develop a wide range of powerful "bubble schedulers", a few examples are provided below.

Burst Scheduling A first example is our previously-described "burst algorithm" 10]: a Self-Scheduling algorithm "pulls" towards processors the bubbles, which "burst" (i.e. release their content on a runqueue) in an opportunistic way 
the machine. The result are shown on Figure 3 , where three busy-looping gangs are sharing a four-processor machine (gang 0 has 5 threads [0-4], etc.)

Work-Stealing Scheduling One of our currently in-progress algorithms is based on work stealing: the hierarchy of bubbles is first settled on the list of processor 0 . Then, when bubble_schedule () is called on an idle processor, it will use our helper functions to look for work to steal locally (on the runqueue of the other processor of the same chip for instance), then more globally, until finding work to steal. The actual work "steal" is a non-trivial algorithmic problem: only a part of the bubble hierarchy should be pulled toward the idle processor, and the structure of the hierarchy should be taken into account as much as possibleAll attributes and statistics attached to bubbles should be carefully taken into account in heuristics, so as to get a distribution suited to the application. These are only purely algorithmic issues though: no technical problems remain.

\section{Implementation}

Our BubbleSched platform is currently implemented as an extension of the MARCELportable two-level thread library with a low additional overhead ( $\sim 5 \%$ on context switches [10]). This library uses operating system functions to detect the machine architecture, bind one kernel-level thread to each processor and it then performs fast user-level context switches between user-level threads. Therefore, assuming no other application is running, it keeps complete control over thread scheduling on processors in userspace. Operations on bubbles can hence be also done in user space, and "Daemon threads" are just MARCEL threads.

Our BubbleSched platform also includes a debugger to help understanding the behavior of a scheduler. A lightweight trace of events (thread birth, sleep, wake up, bubble placement) is recorded during the execution of the application [2] and analyzed off-line. This trace can be converted into an animated movie that shows interactively the series of scheduling events and placement decisions that occurred during the execution. Thus, programmers can easily replay the scheduling decisions at will to find out their algorithmic flaws.

\section{Evaluation}

We show the usefulness of a programmable scheduling platform by introducing an example that illustrates well the dilemma between distributing threads over the machine or keeping related threads close together. It was run on a bi-dualcore Opteron system whose NUMA factor between the dual-cores is around 1.4.

We experimented with SuperLU_MT, a thread-parallel solver for large, sparse systems of linear equations (LU factorization). Figure 1 first shows how well SuperLU scales on the target machine. The speedup is quite close to perfect, up to the number of processors, but because of cache and synchronization affinities, using a number of threads greater than the number of processors (4) just makes 
performances really bad when using generic schedulers like NPTL (LINUX 2.6.17) or original Marcel with a single shared runqueue for all processors.

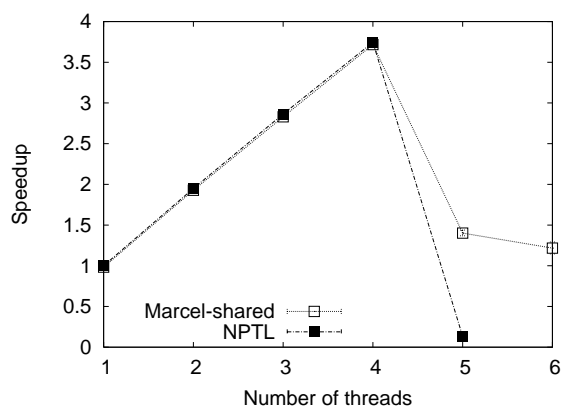

Fig. 4. Parallel speedup of a single job

We consider a situation where a multi-scale scientific application needs to perform LU factorizations jobs "on demand". There are many ways to process these jobs, depending on how many threads to run per job and how to schedule them. Using a mere batch scheduler (running each job 4-way up to completion), or a completely distributed scheduler (running each job 1-way up to completion) may not be wise, in case other parallel parts of the application (running on other machines) need the job result sharply.

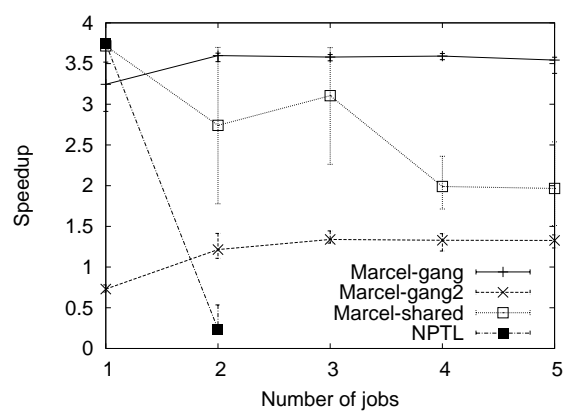

(a) 4-way jobs

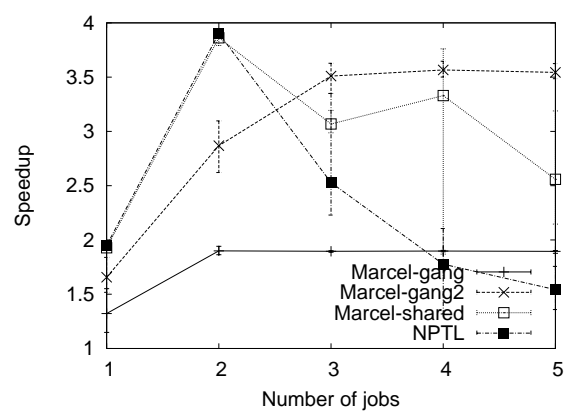

(b) 2-way jobs

Fig. 5. Parallel speedup of mixed jobs, using generic or gang scheduling policies

Figures $5(\mathrm{a})$ and $5(\mathrm{~b})$ show the results obtained using several approaches. On figure 5(a), all jobs were run by using four threads, while on figure 5(b), all jobs 
were performed by using two threads. Both figures clearly show that the generic schedulers of NPTL and original Marcel get very bad and erratic performances (min-max bars are very tall), because they equally process all threads of all jobs without taking affinities into account. We used our gang scheduler of section 3.2 for running the jobs with a time slice of $0.2 \mathrm{~ms}$ (the solving time of one job with only one thread is around 10s) and a single gang scheduler over the whole 4-way machine ("Marcel-gang" curve), but we also tried executing two gang schedulers, one on each 2-way NUMA node of the machine ("Marcel-gang2" curve).

The bottom curve of Figure 5(b) shows that running only one gang scheduler for 2-way jobs obviously limits the speedup to a value of 2 . The bottom curve of Figure 5(a) shows that running 4-way jobs on 2-way NUMA nodes also get a limited speedup (because there are more threads than processors). The top curve of Figure 5(a) shows that our gang scheduler performs quite well at running jobs on the machine: each job seems to achieve a speedup of approximately 3.5, whatever how many there are. The "Marcel-gang2" curve of Figure 5(b) shows that the two gang schedulers achieve this quite well too, provided that there are several jobs, of course. Finally, by carefully comparing these last two curves, one can notice that for this application on this machine, running one gang scheduler for the whole machine is actually a little better than running two separate gang schedulers on each NUMA node. This is probably because we chose, for repartition reasons, to have these two gangs take and put back jobs to a common job pool. Had the application been a little different (less cache- but more memory-bound), we would have noticed the converse.

It must be noted that this experiment was conducted without modifying the application at all. We just made Marcel build bubbles according to natural thread creation affiliation: since threads that work on the same job are created by the same thread, the resulting bubble hierarchy naturally maps to the job. It was then just a matter of starting gang schedulers with appropriate parameters.

\section{Related Work}

Bossa [1] provides scheduling abstractions and a language for developing schedulers. However, its goal is to prove the correctness of the scheduler, and as a such the proposed language, though powerful enough for implementing the LiNUx 2.2 mono-processor scheduler, is quite restrictive and limits programmers a lot.

ELITE [8] provides an a user-scheduling platform taking into account affinities between threads, cache and data. However, it lacks interaction with the application: affinities are detected rather than provided by programmers.

Operating Systems have fairly good schedulers, and Fedorova [4] worked on cache-aware schedulers for Operating Systems. However, these are targetted towards "blind" multi-application situations and hence can't benefit from knowledge provided by the programmer of a scientific application.

Several operating systems provide facilities for distributing kernel threads along the machine by grouping them into sets: liblgroup on Solaris, NSG on Tru64 and libnuma on Linux. These look very much like single level bubbles, but 
no possibility of nested sets is provided, which limits the affinity expressiveness. Moreover, none of them provides the degree of control that we provide: with BubbleSched, the application has hooks at the very heart of the scheduler to react to events like thread wake up or processor idleness.

\section{Conclusion}

In this paper, we present the BubbleSched platform, a tool for designing and prototyping specialized schedulers for specific application domains and libraries (e.g. adaptive mesh refinement algorithms, SPMD codes) for which threads behavior and memory affinities can be predicted to some extent. It provides programmers both with a way to express the application structure, and several high-level scheduling distribution primitives that let scheduling experts write bubble algorithms that tightly "drive" the thread scheduler by implementing some hooks. Examples of implementing scheduling strategies have shown how easy this is and how powerful it can be. Non-expert programmers may try different combinations of existing strategies to schedule threads, focusing on algorithmic issues rather than on gory details. Actually, part of this work was done in collaboration with researchers at the CEA (french Atomic Energy Commission) who have been developing huge HPC applications for a few decades and who are looking for a tool allowing them to transfer their expertise to the underlying runtime system. The conduction-advection application described in the paper is an example of such application where we have demonstrated the interest of our approach.

This work opens lots of future prospects. In the short term several algorithmic approaches will be tested and tuned to schedule real applications. On a longer run, a generic tunable scheduler could take into account as much information as possible from the hardware, the compiler and programmers. An integration to the LINUX kernel could even be considered, since a bubble hierarchy naturally exists through the notions of threads, processes, sessions and users.

\section{References}

1. L. P. Barreto and G. Muller. Bossa: une approche langage à la conception d'ordonnanceurs de processus. In Rencontres francophones en Parallélisme, Architecture, Système et Composant (RenPar 14), Hammamet, Tunisie, Apr. 2002.

2. V. Danjean, R. Namyst, and P.-A. Wacrenier. An efficient multi-level trace toolkit for multi-threaded applications. In EuroPar, Lisbonne, Portugal, Sept. 2005.

3. D. Durand, T. Montaut, L. Kervella, and W. Jalby. Impact of memory contention on dynamic scheduling on NUMA multiprocessors. In Int. Conf. on Parallel and Distributed Systems, volume 7. IEEE, Nov. 1996.

4. A. Fedorova. Operating System Scheduling for Chip Multithreaded Processors. PhD thesis, Harvard University, Cambridge, Massachusetts, 92006.

5. P. Hénon, P. Ramet, and J. Roman. PaStiX: A parallel sparse direct solver based on a static scheduling for mixed $1 \mathrm{~d} / 2 \mathrm{~d}$ block distributions. In Proceedings of the 15 IPDPS 2000 Workshops on Parallel and Distributed Processing, Jan. 2000. 
6. J. Marathe and F. Mueller. Hardware profile-guided automatic page placement for ccnuma systems. In Sixth Symposium on Principles and Practice of Parallel Programming, Mar. 2006.

7. X. Shen, Y. Gao, C. Ding, and R. Archambault. Lightweight reference affinity analysis. In 19th ACM International Conference on Supercomputing, pages 131140, Cambridge, MA, USA, June 2005.

8. M. Steckermeier and F. Bellosa. Using locality information in userlevel scheduling. Technical Report TR-95-14, University of Erlangen-Nürnberg, Dec. 1995.

9. S. Thibault. BubbleSched API. http://runtime.futurs.inria.fr/marcel/doc/.

10. S. Thibault. A flexible thread scheduler for hierarchical multiprocessor machines. In Second International Workshop on Operating Systems, Programming Environments and Management Tools for High-Performance Computing on Clusters (COSET-2), Cambridge / USA, 06 2005. ICS / ACM / IRISA. 